\title{
Configurational Temperature and Monte Carlo Simulations
}

\author{
A.C. Brańka ${ }^{1}$, S. Pieprzyk ${ }^{2}$ \\ ${ }^{1}$ Institute of Molecular Physics, Polish Academy of Sciences, \\ ul. M. Smoluchowskiego 17, 60-179 Poznań, Poland \\ e-mail:branka@ifmpan.poznan.pl \\ ${ }^{2}$ Technical Physics Faculty, Poznań University of Technology, \\ ul. Nieszawska 13a, 60-965 Poznań, Poland
}

(Received: 15 October 2010; revised: 16 November 2010; accepted: 26 November 2010; published online: 8 December 2011)

\begin{abstract}
Two configurational temperature expressions are investigated from the point of view of their utility as a diagnostic tool for Monte Carlo (MC) simulations. The MC calculations were performed for systems of spherically symmetric particles in the bulk and in a channel. Different density and system size conditions are considered. It is shown that the configurational temperature based on the ratio of two averages of the force functions can serve as a suitable method for calculating the temperature for the MC simulation. This configurational temperature can signal the presence of random number correlations and can serve as a cross-check formula in $\mathrm{MC}$ studies of strongly confined systems.
\end{abstract}

Key words: temperature, configurational temperature, computer simulations, Monte Carlo method, confined systems

\section{INTRODUCTION}

The ability to control or monitoring temperature of simulated systems is an important issue for many techniques. In deterministic simulations such as molecular dynamics, the calculation of temperature until relatively recently has been based almost exclusively on the equipartition theorem which expresses temperature as the average kinetic energy per degree of freedom of the system. This temperature is traditionally called the kinetic temperature, $T_{K}$. In stochastic simulations carried out with Monte Carlo or Brownian Dynamics methods, for example, only positions or configurations of the system are generated and therefore the kinetic temperature cannot be calculated. In this case the temperature may be an input quantity, which cannot therefore be verified directly within the simulation. Also, for systems that are away from equilibrium, temperature control based on the kinetic temperature definition is problematic as in general its profile is not known a priori or unambiguously $[1,2]$.

An important turning point in the field of temperature control was initiated by the work of Rugh [3]. Starting from the thermodynamic relation of temperature, $1 / T=\partial S / \partial E$ Rugh derived a new formula for the temperature,

$$
\frac{1}{k_{B} T_{R}}=\left\langle\nabla \cdot\left[\frac{\nabla H(\Gamma)}{(\nabla H(\Gamma))^{2}}\right]\right\rangle,
$$

where the angular brackets denote a microcanonical ensemble average, $H=K(\mathbf{p})+U(\mathbf{q})$ is the system's Hamiltonian, and $\nabla$ involves all the degree of freedom of the system $\Gamma=(\mathbf{p}, \mathbf{q})=\left(p_{1}, p_{2}, \ldots p_{3 N}, q_{1}, q_{2}, \ldots q_{3 N}\right) . K$ and $U$ are kinetic and potential energy, respectively. The formula given in Eq. (1) led to various expressions for the temperature which are equivalent in the large system limit. The most general form was proposed by Butler et al. [4] and Rickayzen and Powles [5]

$$
k_{B} T=\frac{\langle\nabla H(\Gamma) \cdot \mathbf{B}(\Gamma)\rangle}{\langle\nabla \cdot \mathbf{B}(\Gamma)\rangle} .
$$

The attractive feature of Eq. (2) is that $\mathbf{B}(\Gamma)$ can be any continuous and differentiable vector in phase space. In the case, $\mathbf{B}(\Gamma)=\left(p_{1}, p_{2}, \ldots, p_{3 N}, 0,0, \ldots, 0\right)$ Eq. (2) yields the equipartition theorem and the familiar kinetic tem- 
perature is obtained, $k_{B} T_{K}=\left\langle(1 / N) \sum p_{i}^{2} / 2 m\right\rangle$, where $m$ is the mass of the particle. Discussion on different analytic forms for temperature based on this general expression can be found in Refs. [6-8] and some of the main formulae which can be derived from Eq. (2) for different choices of $\mathbf{B}(\Gamma)$ are shown in the schematic diagram in Fig. 1.

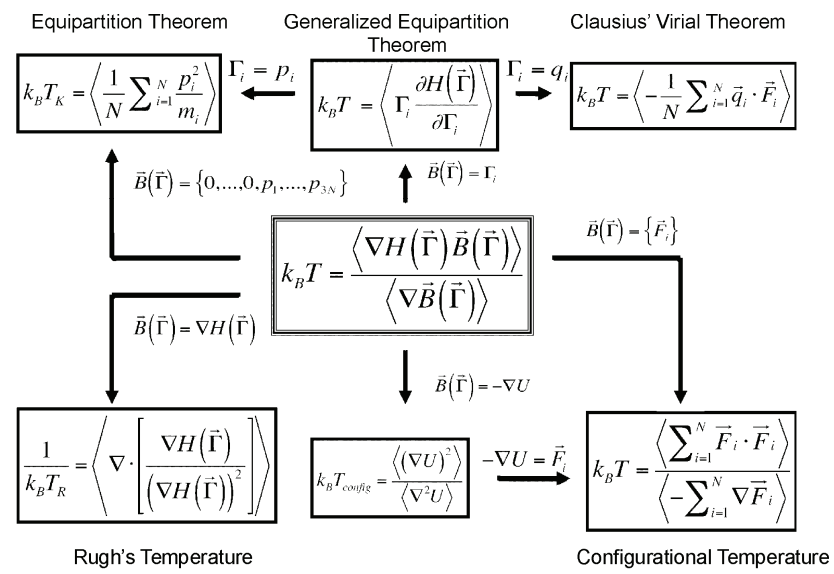

Fig. 1. Various temperature expressions derived from the most general (central) formula are given in the boxes. Different choices for the phase space vector $\mathbf{B}(\Gamma)$ used in derivation are shown

Among the different expressions for the temperature, a particularly important example is that following from the choice $\mathbf{B}(\Gamma)=-\nabla U$. In this case the temperature can be written as,

$$
k_{B} T_{\text {config }}=\frac{\left\langle(\nabla U)^{2}\right\rangle}{\left\langle\nabla^{2} U\right\rangle} .
$$

Note that in the above expression, the temperature depends only the particle positions and not on their momenta, so it is possible to determine the system's temperature from the configurations. The temperature in Eq. (3) has naturally been called the configurational temperature [4]. This new possibility is illustrated in Fig. 2. The configurational temperature broadens our understanding of the meaning of 'temperature', as a physical property, as it is now calculable in a simulation from other quantities apart from the particle momenta, the traditional choice. It has opened up new possibilities to calculate the temperature and investigate problems where the kinetic temperature is not otherwise accessible. Examples include, a new scheme to measure pair potentials in soft-matter systems [7], new thermostats for atomic and molecular systems at equilibrium and in steady states [8], and as a diagnostic tool to check the correct implementation of algorithms in stochastic method computer codes $[4,9]$.

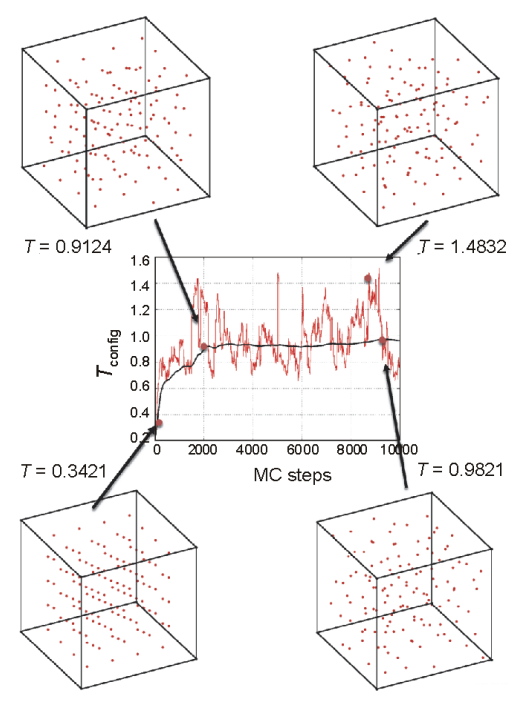

Fig. 2. Illustration of the configurational temperature as a function of the number of steps. The thin line represents the instantaneous temperature $\left((\nabla U)^{2} / \nabla^{2} U\right)$ of successive configurations produced in the MC simulations of system of $N$ particles in the constant volume $V$ at temperature $T=1$. Four selected configurations are drawn (note the crystalline initial configuration yields very low instantaneous temperature and the very disordered configuration is one of the highest temperature or 'hottest' configurations). The bold solid line is the average over instantaneous temperatures and it tends towards the set in simulations value, $T=1$

In this work we concentrate on two features of the configurational temperature which are important for MC simulations. The first is its usefulness in checking that the actual temperature of the system is the same as the 'input' temperature in the MC code. The second is in the reliability of the configurational temperature expressions for confined systems. It has been demonstrated that the $O(1 / N)$ difference between different expressions for configurational temperature varies with density and system size [10]. The results obtained for model fluids in a narrow slit pore geometry with periodic boundaries demonstrated a different system-size dependence of the various temperature expressions, as well as the utility of Eq. (3) [9]. In strongly confined systems, such as where the particles are in a microchannel, the presence of the walls does influence the behaviour of the system and the system-size dependence of the various configurational temperatures. The aim of this work is to obtain a quantitative assessment of this dependence.

\section{MC SIMULATION}

By making different choices for $\mathbf{B}(\Gamma)$, various expressions for the configurational temperature can be derived 
which differ in form and numerical efficiency. Some of these can be exploited as a diagnostic tool for the MC simulations. The requirement for any such expression is that it should have a relatively simple analytic form, be easily implemented in a simulation code and have as small a system size dependence as possible. In what follows we concentrate on two configurational temperature expressions defined through the definition,

$$
B=-\nabla U=\left(\mathbf{F}_{1}, \mathbf{F}_{2}, \ldots \mathbf{F}_{N}, 0,0, \ldots, 0\right),
$$

where

$$
\mathbf{F}_{i}=-\nabla_{i} \sum_{j \neq i}^{N} u\left(r_{i, j}\right),
$$

is the force acting on the $i$ th particle and $u$ is a pairwise additive potential. They are denoted by $T_{\text {conf } 1}$, and $T_{\text {conf } 2}$ with the definitions,

$$
\begin{aligned}
& k_{B} T_{\text {conf } 1}=\frac{\left\langle\sum_{i=1}^{N} \mathbf{F}_{i}^{2}\right\rangle}{\left\langle-\sum_{i=1}^{N} \nabla_{i} \mathbf{F}_{i}\right\rangle}, \\
& k_{B} T_{\text {conf } 2}=\left\langle\frac{\sum_{i=1}^{N} \mathbf{F}_{i}^{2}}{-\sum_{i=1}^{N} \nabla_{i} \mathbf{F}_{i}}\right\rangle
\end{aligned}
$$

Note that $T_{\text {conf } 2}$ is the average of one q-dependent function and $T_{\text {conf } 1}$ is a ratio of two averages of such quantities.

To assess the performance of the above configurational temperature expressions, calculations were performed for relatively small systems and two different types of particle assembly were considered. In the first, a bulk fluid was studied composed of $N$ particles interacting via the inverse power pair potential $u$, with periodic boundary conditions (p.b.c.) in all three cartesian directions. In the second, the same system was placed into a channel geometry (with volume, $V=L \times L \times 2 L$ ) of square cross-section and a basic side-length, $L$, of a few particle diameters dimension. Along the channel the p.b.c. were applied, as illustrated in Fig. 3.

The inverse-power system is one in which the particles interact via the pair potential,

$$
u(r)=\varepsilon\left(\frac{\sigma}{r}\right)^{n},
$$

where $r$ is the separation between the centres of two particles, $\sigma$ is a nominal particle diameter, $\varepsilon$ establishes the energy scale, and $n$ controls the stiffness of the potential (the softness is $\sim 1 / n$ ). This potential has the useful feature that excess thermodynamic quantities of the inverse-power systems are functions only of the quantity $\rho^{*}=\left(\varepsilon / k_{B} T\right)^{3 / n} \rho \sigma^{3}$ where $\rho=N / V$ is the number density, for $N$ particles in volume $V$ [11].

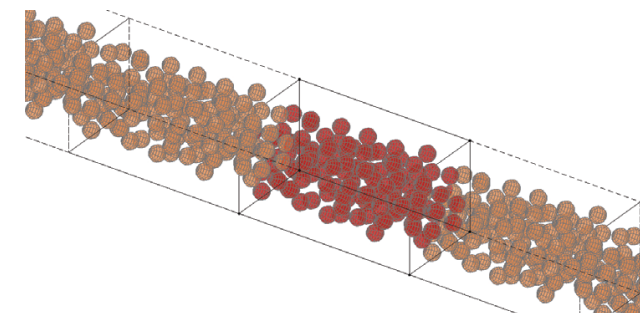

Fig. 3. The channel geometry used in this work. The main MC cell contains $N$ particles and its size is $L \times L \times 2 L$. Particles in this cell are drawn darker. The periodicity direction is $z$-direction

In this study, $n=12$ and the reduced temperature, $T^{*}=k_{B} T / \varepsilon=1$ was used. The NVT Monte Carlo [2, 12] calculations were performed at three representative fluid densities $\rho^{*}=0.3,0.5$ and 0.7 . For each density the system size dependence was studied for $N=35,62,128$ and 275. At each $\rho^{*}$ and $N$, after a long equilibration simulation, the averages were calculated from simulations of $10^{6} \mathrm{MC}$ steps (the acceptance ratio was kept at the level of 50 percent). It was verified that the accuracy of the calculated quantities was sufficient for the purposes of this work. In what follows all quantities are given in terms of the basic units $\sigma, \varepsilon$ and $m$, e.g., $U^{*}=U / \varepsilon$ and the asterisk is omitted.

In the channel geometry the interaction between the particles and the (smooth) channel walls, $u_{W}(r)$ was modeled by the same inverse power interaction, given in Eq. (7), but with $n=36$, and now $r$ is the distance of the particle from the channel wall. Such a strongly repulsive interaction for the confining walls only marginally contributes to the total energy but is have to be included in the $T_{\text {config }}$ calculations.

\section{III. $T_{\text {conf }}$ AS DIAGNOSTIC TOOL IN BULK SYSTEMS}

The system-size dependence of the configurational temperatures at different densities for the bulk systems are shown in Fig. 4. At all densities the $N$-dependence is seen to be well- 
represented by the linear scaling $T(\infty)=T(N)+\alpha / N$ for $T_{\text {conf } 1}$ and $T_{\text {conf } 2}$. However, the slope $\alpha$ is different for these configurational temperature expressions. In the case of $T_{\text {conf } 2}$ the slope is significant and changes with density. Consequently, in the MC code, the input thermodynamic temperature $T=1$ can be verified conclusively via linear extrapolation after performing simulations for at least two different numbers of particles, $N$. In the case of $T_{\text {conf } 1}$, the slope is close to zero at all densities which means that this expression can be exploited as the temperature control tool even in the case of relatively small systems. These findings support previous observations made for other systems [10].
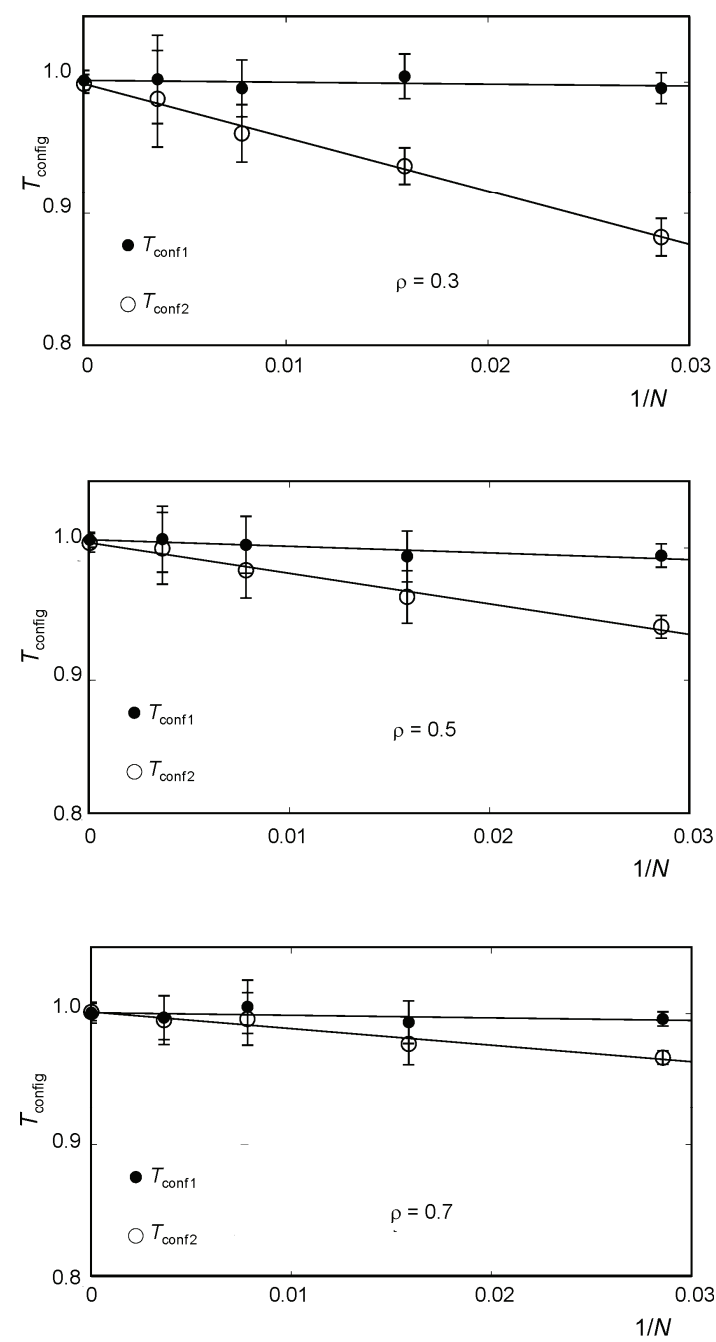

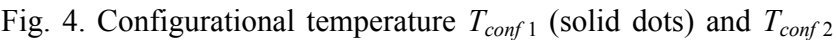
(open circles) versus system size for bulk systems. The results for the inverse-power fluid at three densities are given in the figures

Next, the ability of the $T_{\text {conf } 1}$ formula to signal errors in calculated properties connected with correlations among random numbers was tested. Such correlations may be easily be introduced into a computer code simply as a typographical error, by repeating the name of a stochastic variable, or by the inappropriate use of the random number generator. We stress that there are no simple rules to identify such kind of errors in the MC programs, which is why the configurational temperature can offer a readily incorporated useful check on the implementation of a particular MC scheme. In Fig. 5 results of two MC simulations are shown, one without random number correlations and one in which the particle random displacements in $X$ and $Y$ directions are constrained to be the same at every third MC step (i.e. every three one particle attempted moves)
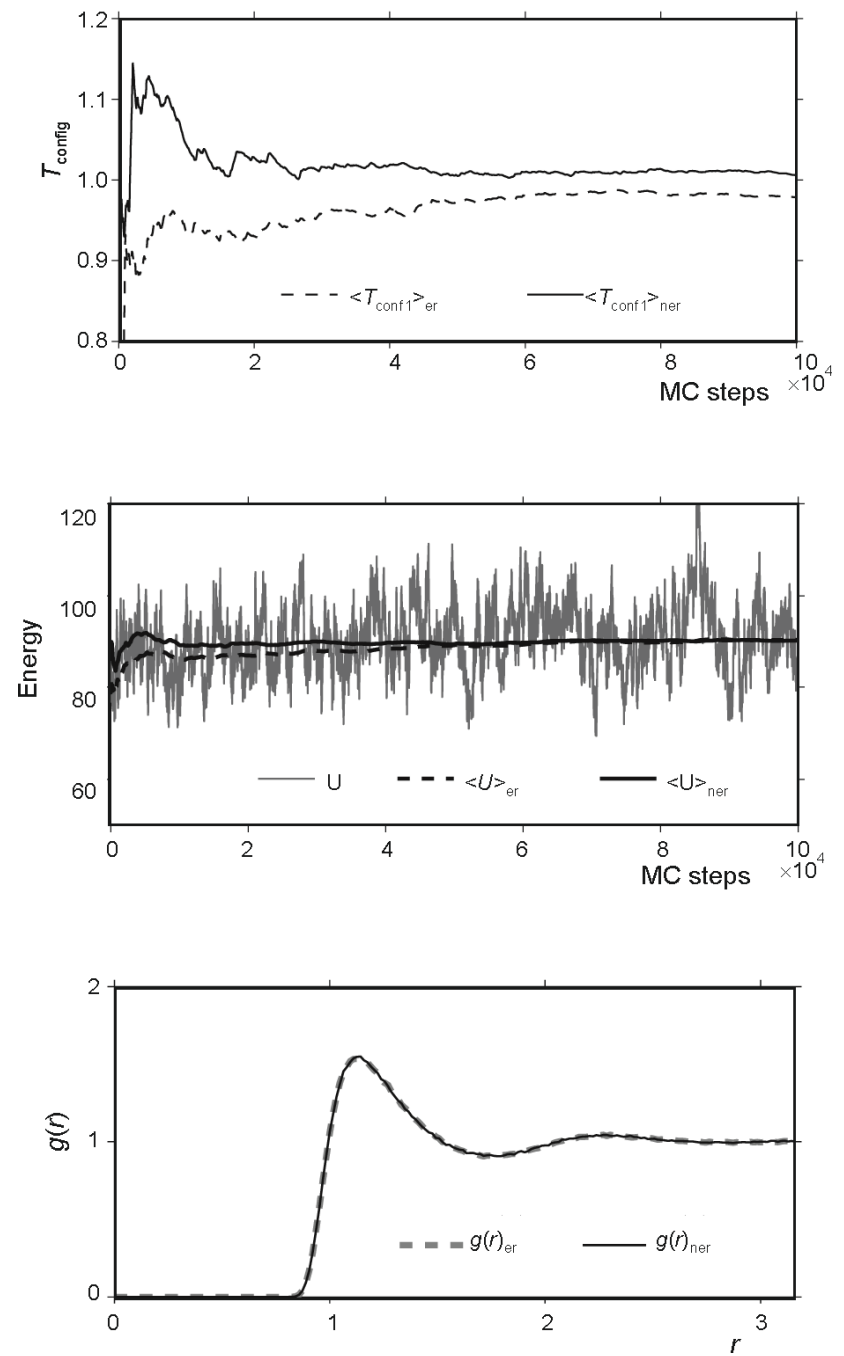

Fig. 5. The configurational temperature $T_{\text {conf } 1}$, total energy and the radial distribution function (from top to bottom) of the bulk inverse-power fluid at $\sigma=0.5$ and $N=128$. Results obtained from the MC simulation with uncorrelated and correlated random numbers are denoted with the index 'ner' and 'er' respectively. The instantaneous total energy $U_{e r}$ and $U_{n e r}$ are indistinguishable and are denoted by $U$ 
As is clearly visible in the figure, the basic physical quantities such as total energy $U$ and the radial distribution function $g(r)$ are practically the same in both cases (similar behaviour is obtained for the pressure). Thus, the introduced error (i.e., correlation between random numbers) is 'invisible' in most calculated in the MC simulation physical quantities. This subtle type of error shows up clearly in the configurational temperature, however. As seen in Fig. 5, the error-free simulation yields the expected value of $\left\langle T_{\text {conf } 1}\right\rangle_{\text {ner }} \cong 1$. For the same conditions the configurational temperature of system with correlated displacements, $\left\langle T_{\text {conf } 1}\right\rangle_{e r}$, quickly reaches its ultimate value which is, however, lower than the correct value of $T=1$. The above test demonstrates the usefulness of the $T_{\text {conf } 1}$ formula as an invaluable cross-check for the MC simulations.

\section{IV. $T_{\text {conf }}$ IN CONFINED SYSTEMS}

It is known that the physical properties of a confined system are generally quite different from its bulk properties
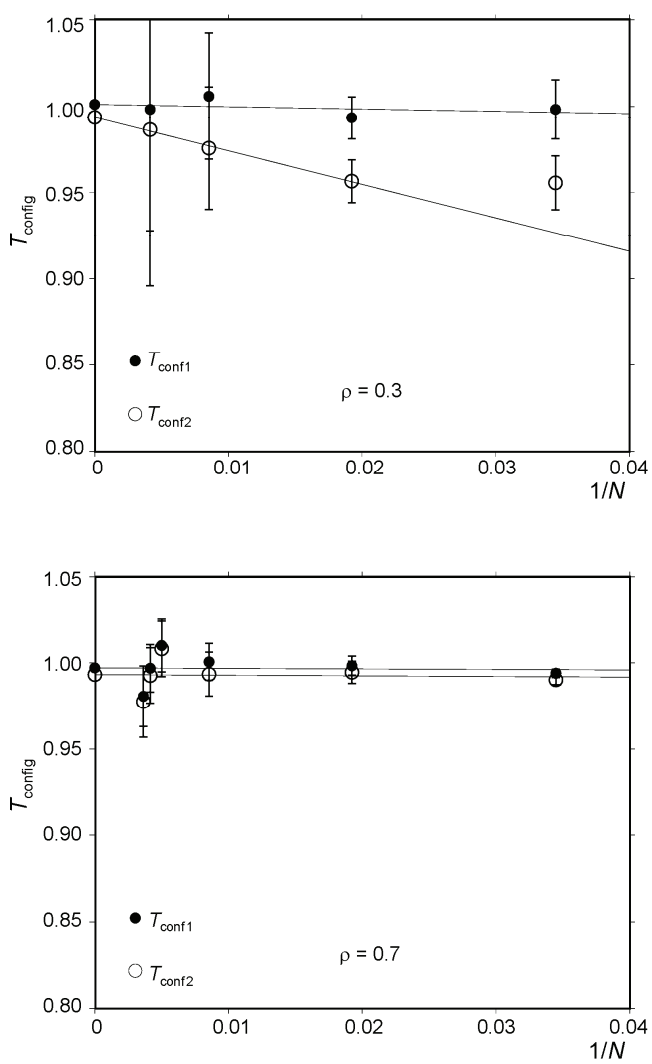

at the same (or as similar as possible) state points. In the previous section, the configurational temperature of the bulk system was considered. In this section the influence of a strong system confinement on this property is discussed. An important question is, can the configurational temperature also be a useful diagnostic tool for situations where the system is strongly confined in two spatial directions. In Fig. 6 results are shown for $T_{\text {conf } 1}$ and $T_{\text {conf } 2}$ of the inversepower system in the channel geometry illustrated in Fig. 3. Calculations were performed at four densities (the same as in the bulk case) and an additional one at $\rho=0.9$. There are apparent similarities between these results and the bulk case results shown in Fig. 4.

As in the bulk case, the advantage of $T_{\text {conf } 1}$ over $T_{\text {conf } 2}$ is evident. The density trend of the linear slopes in both cases is similar but, in the channel geometry the $T_{\text {conf } 2}(1 / N)$ reaches the linear character only for $N>100$. Thus, $T_{\text {conf } 2}$ should not be used as the temperature assessment tool for strongly confined systems where one typically deals with a small number of particles. The results demonstrate that $T_{\text {conf } 1}$ can be a suitable direct temperature check also in the case of the strongly confined system in the channel. Note,
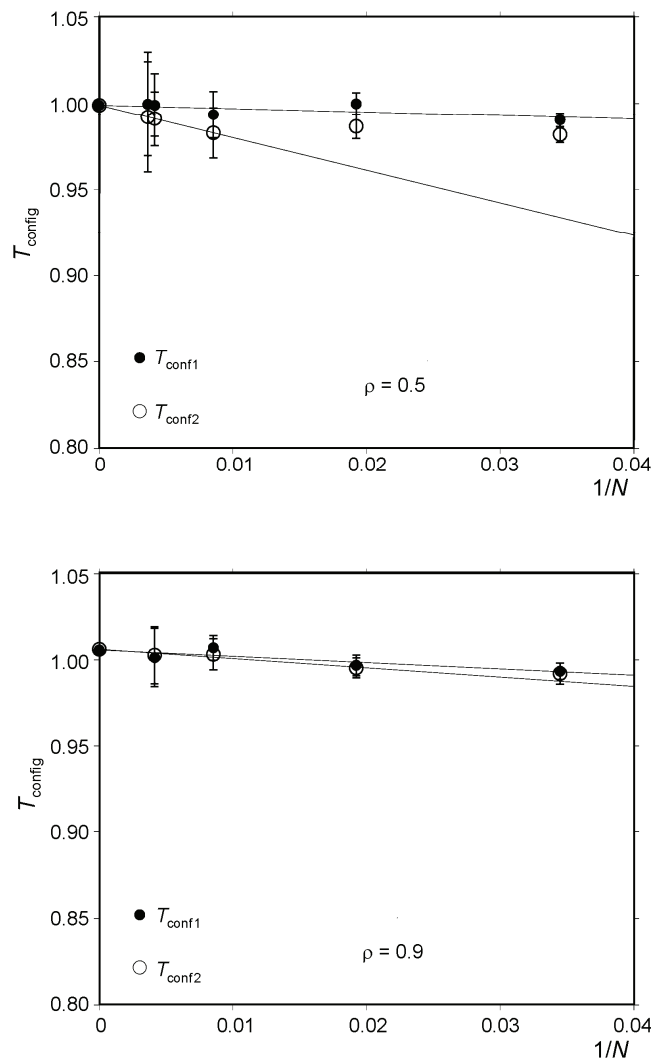

Fig. 6. The configurational temperature, $T_{\text {conf } 1}$ (solid dots) and $T_{\text {conf } 2}$ (open circles) $v s 1 / N$ for the inverse-power fluid in the channel geometry shown in Fig. 3 at four different densities which are given on the figures. The thin solid lines are linear fits (in the fit for the $T_{\text {conf } 2}$ ) the data for the two smallest $N$ were not taken into account) 
that in the situation in which $N<100$ additional simulation segments with different $N$ may be required to determine the slope and the MC temperature. At $\rho=0.7$ the simulations for different $N>100$ produce quite irregular behaviour and the expected target temperature, $T=1$, is hardly obtained
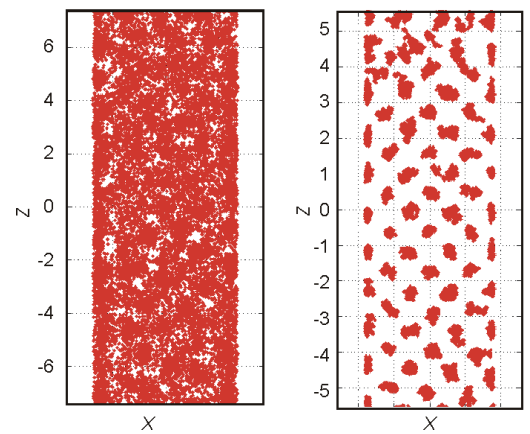

Fig. 7. Particle assembly Structure in the channel at $\sigma=0.3$ (left figure) and at $\sigma=0.7$ (right figure). The figures show the projections of $10^{4}$ configurations on the $Z X$ plane. A liquid-like region in top part of the right figure indicates the system is in the transition state

within statistical uncertainly. An inspection of the structural quantities, $g(r)$ and the one-particle distribution given in Fig. 7, reveals that at this density a transition from a liquid-like to a solid-like structure takes place in the system. The new temperature expressions outlined in the diagram in Fig. 1 have been derived within the framework of the equilibrium state statistical mechanics and are therefore not necessarily valid under phase transition conditions. Thus, the slight departure from the MC temperature at $\rho=0.7$ can be understood, in fact, as a confirmation of this limitation. At $\rho=0.9$ the system is in the solid-like phase and in this case both expressions for the configurational temperature produce a very similar $1 / N$-dependence.

\section{SUMMARY}

In this work, two configurational temperature expressions, $T_{\text {conf } 1}$ and $T_{\text {conf } 2}$, were investigated from the point of view of their possible utility as a diagnostic tool for the MC simulations. Both expressions are combinations of the particle forces and their derivative. The test system was composed of particles interacting via a repulsive inverse power potential, and NVT Monte Carlo calculations were performed for the bulk and a channel geometry at several densities and different system sizes. It was found that in all cases studied, $T_{\text {conf } 1}$ hardly depended on the system size, $N$. Unlike $T_{\text {conf } 1}$, the temperature, $T_{\text {conf } 2}$ displays a considerable $N$-dependence and in the case of strongly confined small systems (i.e., $N<100$ in the channel geometry) this expression was practically useless as a measure of the temperature in the MC simulation. It was also discovered that $T_{\text {conf } 1}$ is particularly sensitive to introduced random number correlations. Our results indicate that the configurational temperature $T_{\text {conf } 1}$ is a valuable diagnostic tool for MC simulations. In particular, it can be used to verify the $\mathrm{MC}$ temperature, and used to detect subtle stochastic errors connected with correlations between random numbers. This relatively simple expression is well worth implementing into an MC code, and can significantly reduce the risk of carrying out inaccurate implementations of a given stochastic simulation technique (typically Monte Carlo or Brownian Dynamics).

\section{Acknowledgments}

The authors are grateful to Prof. David Heyes for helpful remarks. The work has been partially supported by the Polish Ministry of Science and Higher Education grant N N202 261438.

\section{References}

[1] J.-P. Hansen, I.R. McDonald, Theory of Simple Liquids, 2005, 3rd. ed. Academic Press, Amsterdam.

[2] M.P. Allen, D.J. Tildesley Computer Simulation of Liquids, Oxford University Press: 1987.

[3] H.H. Rugh, Phys. Rev. Lett. 78, 772 (1997).

[4] B.D. Butler, G. Ayton, O.G. Jepps, D.J. Evans, J. Chem. Phys. 109, 6519 (1998).

[5] G. Rickayzen, J.G. Powles, J. Chem. Phys. 114, 4333 (2001).

[6] J.G. Powles, G. Rickayzen, D.M. Heyes, Mol. Phys. 103, 1361 (2005).

[7] Y. Han, D.G. Grier, J. Chem. Phys. 122, 64907 (2005).

[8] K.P. Travis, C. Braga, J. Chem. Phys. 128, 14111 (2008).

[9] J. Delhommelle, D.J. Evans, J. Chem. Phys. 114, 6236 (2001).

[10] O.G. Jepps, G. Ayton, D.J. Evans, Phys. Rev. E 62, 4764 (2000).

[11] Y. Hiwatari, H. Matsuda, Pog. Theor. Phys. 47, 741 (1972).

[12] N. Metropolis, A.W. Rosenbluth, M.N. Rosenbluth, A.H. Teller, E. Teller, J. Chem. Phys. 21, 1087 (1953). 


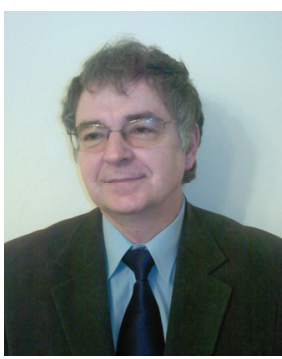

Akadiusz Brańka, Prof. IFM PAN, studied physics at the Adam Mickiewicz University in Poznań. He obtained his $\mathrm{PhD}$ in ISAS in Trieste in 1986 on the subject of computer simulation methods under the supervision of Professor Michele Parrinello. After his $\mathrm{PhD}$ joined the Institute of Molecular Physics Polish Academy of Science, where in 2002 he received habilitation. His interest are in the structural, thermodynamics and transport properties of liquids and suspensions, anomalous elasticity in solids and simulation methods with particles.

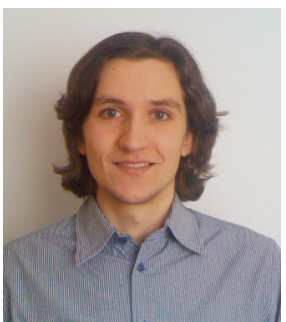

SŁawomir PiePrzyk, was born in Wolsztyn in 1986. He received M.Sc. degree in technical physics in 2010 from Poznan University of Technology. During the studies he was trained in algorithms for industrial robots. He completed his master thesis in IFM PAN. His research interest involve object-oriented programming and computer simulations of particle systems. 